\title{
Analysis of some problems in classification of seabed bottom characteristics using acoustic backscattering intensity
}

\author{
FENG Jintao $^{1}$, BAO Jingyang ${ }^{2}$, JIN Shaohua $^{1}$, XIAO Fumin ${ }^{1}$, BIAN Gang ${ }^{1}$ \\ ${ }^{1}$ Department of Military Hydrographic Surveying and Charting, Dalian Naval Academy, Dalian, Liaoning \\ 116018 \\ ${ }^{2}$ School of Geodesy and Geomatics, Wuhan University, Wuhan 430079, China
}

\begin{abstract}
The backscattering intensity collected by multi beam sonar system and scanning sonar system can be used to classify seabed bottom characteristics. However, there are many problems that have not been solved in the practical application. This paper attempts to sort out and analyze the problems encountered in the classification process and put forward some solutions. The relationship among acoustic image, seabed topography and sediment is analyzed
\end{abstract}

\section{Introduction}

SSS (Side-scan Sonar System) and MBS (Multi-beam Bathymetric System) not only provide deep water information, but also carry sound intensity data back. The reflected intensity of sound intensity signal on the seafloor is related to the bottom geology and topography.Sound map, seafloor topography and seabed bottom characteristics are three very important basic geographic information. They correspondingly reflect the texture, fluctuations and seabed bottom characteristics composition of the seafloor. They are important elements for us to understand and utilize the ocean, and are also hot issues in the world.And the bottom geology and terrain have a deep influence on the echo intensity. That is, the echo intensity contains the information of seabed bottom characteristics, so we can classify the seabed bottom characteristics, which undoubtedly improves the efficiency of classification compared with the method of submarine sampling. It can not only retrieve the type of seabed bottom characteristics, but also draw the boundary of different types of seabed bottom characteristics conveniently, thus greatly reducing the project cost.Sufficient research has been carried out at home and abroad on this issue, but many bottlenecks have been encountered at present.

(a) What is the relationship between the distribution of echo intensity and seabed bottom characteristics.

(b) The classification accuracy of seabed bottom characteristics based on sonar image is low.

(c) Seabed bottom characteristics classification directly below the transducer and in the shaded and radiation residual areas of the target image

(d) Deep study on the classification of large area seabed bottom characteristics. (e) How the echo intensity only retains the information of seabed bottom characteristics and strips the influence of topographic information

Therefore, it is necessary to study the submarine reflection model of sound wave, the response mechanism of submarine topography and seabed bottom characteristics to echo intensity and their contribution and separation.And break through the bottleneck of restricting large area, high accuracy and high efficiency geomorphic image acquisition, terrain inversion and seabed bottom characteristics classification. Achieve the integrated acquisition of submarine geomorphic image, terrain and seabed bottom characteristics information based on acoustic intensity echo information.

\section{Development of Marine Acoustic Equipment for seabed bottom characteristics Detecting}

At present, the subsea topography, geomorphic image and seabed bottom characteristics are mainly obtained by means of multi-beam (MBS), side-sweep sonar (SSS) and subsea sampling. MBS can sweep the subsea in full coverage, and hundreds of sounding points are obtained by single pulse/ping. Compared with single beam, the braid ratio and efficiency of sounding are significantly improved.In order to improve the sweeping range, the MBS usually uses the isogonal measuring mode. The spacing between the seafloor measuring points increases with the increase of the incident angle of the beam and the water depth, and the braid ratio of the depth measurement in the deep sea is not high. If the accuracy and braid ratio are guaranteed, the sweeping width must be reduced.Therefore, there is still room for improvement in strategy to achieve fast, high accuracy and high braid rate acquisition of seabed bottom characteristics in large area by means of MBS.SSS reflects the change of

whitefjt@163.com 
seafloor texture through line scanning imaging. 600012000 echoes can be obtained by single Ping scanning, which is 60-120 times the braid rate of MBS sounding. However, due to the influence of flamboyant measuring mode, the image position accuracy is low, the azaki change and the lack of elevation information ${ }^{[1]}$.SSS improves the sweeping range significantly compared with MBS, and the closer the SSS is to the seafloor, the better the imaging quality is.Splicing multiple strips of SSS images can achieve fast acquisition of large-area seafloor geomorphic images, but affected by many factors, SSS images are mainly used to find targets, and there is still a gap between fine quantitative expression of seafloor geomorphic features ${ }^{[2,3]}$.Seabed bottom characteristics is mostly acquired by sampling. Accurate information about the seabed bottom characteristics is obtained by laboratory analysis. However, to obtain large area of seabed bottom characteristics with high braid rate, a lot of sampling work needs to be carried out, which has shortcomings of high cost and low efficiency.

The new SSS developed in recent years has the characteristics of multi-frequency, multi-pulse and deep drag measurement, which can approach the seafloor to a greater extent and carry out larger and higher braid ratio seafloor geomorphic sweeping.The synthetic aperture sonar measuring system based on interference principle further improves the imaging braid ratio ${ }^{[4,5]}$. The new SSS trailer is equipped with DVL, INS, compass, pressure sensor, attitude sensor and depth sounder, which can provide accurate position, speed, direction, dragging depth and altitude information for the trailer in real time. To a certain extent, the inaccurate position problem that has puzzled SSS image for a long time in quantifying submarine landform is solved.

\section{Problems in Data Processing}

\subsection{SSS Data Processing}

The main focus of the problem is on the following aspects:

(a) Time-varying gain(TVG) compensation is mostly realized by theoretical model. Because it is difficult to give accurate parameters, compensation is incomplete and radiation distortion is often caused.The beam mode and sound angle response can be compensated by means of intensity-angle curve ${ }^{[6]}$, and the model parameters can be corrected by means of echo intensity/waterfall map spatio-temporal statistical distribution. These studies take into account some radiation distortion inducements and improve the image quality to a certain extent, but do not take into account the bottom quality. After correction, the image quality is still poor.

(b) When tracking the seafloor, only the ideal measurement condition is considered and the seafloor is obtained by gray level mutation. However, when suspended matter exists in the water body, the method fails and the real seafloor line can not be obtained, which affects the spatial quantitative expression of the image on the seafloor. (c) When reconstructing the image in the blank area, ignoring the change of seafloor texture, inserting the blank area directly into the gray scale of neighborhood pixels, which results in the image mosaic problem protruding network.

(d) Due to the influence of beam mode and other factors, the echo intensity just below the trailer changes greatly, which reflects the distortion of seafloor topography, and there is no better solution network at present.

(e) It is difficult to obtain large area and high quality images.Influenced by such factors as low positioning accuracy and distortion, large area image target dislocation and distortion caused by multi-band image splicing is outstanding ${ }^{[7,8]}$. The above problems have puzzled the acquisition of large-area and high-quality geomorphic images for a long time and the accurate and quantified tables of seafloor geomorphic features, which need to be further studied.

\subsection{MBS Data Processing}

Compared with side scan sonar, MBS can provide more data information for seabed bottom characteristics classification, but due to its measuring structure and data acquisition characteristics, the following problems exist:

(a) There are abnormal values of the echo intensity near the central beam (transmitting angle $0 \sim 20$ ), which seriously affect the classification of the seabed bottom characteristics;

(b) Multi-beam sonar image is slightly worse than side-scan sonar image, which is not conducive to display fine texture features of the seafloor.

\section{Relationship between seabed bottom characteristics classification, topography and echo intensity}

The correlation between backscattering intensity and seabed bottom characteristics type is the acoustic theoretical basis for the classification of seabed bottom characteristics. The sea bottom scattering intensity of the measured data at sea indicates that its values are influenced by many factors, including the frequency of sound waves, the grazing angle (residual angle of the bottom incident angle) and the seabed bottom characteristics.The results of the variation of backscattering intensity from different frequencies of sound waves show that the roughness of the seafloor affects the process of bottom acoustic scattering. When the roughness is greater than the wavelength, the backscattering is independent of the frequency, while when a considerable portion of the seafloor roughness is smaller than the wavelength, the backscattering intensity increases with the frequency (Jackson, 1986).Frequencies range from $12 \mathrm{kHz}$ (deep water) to $55 \mathrm{kHz}$ (shallow water), resulting in changes in seafloor backscattering intensity up to $10 \mathrm{~dB}$, which are difficult to remove. When processing the data of seafloor backscattering intensity with different measuring devices, 
attention should be paid to the variation of seafloor backscattering intensity caused by different frequencies between the two.

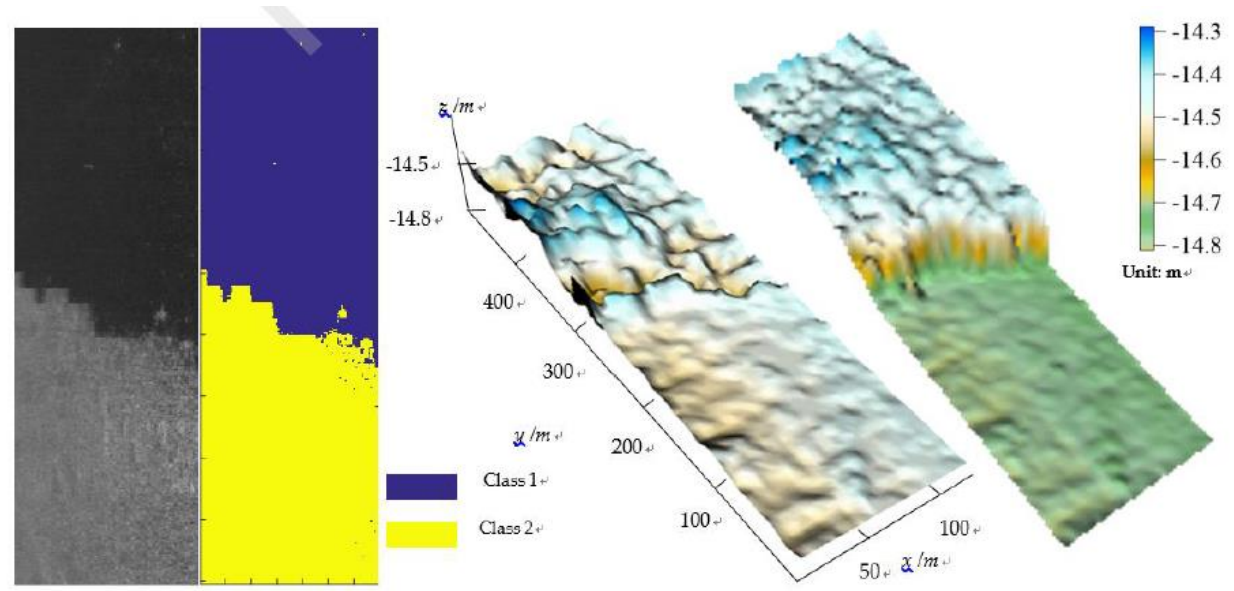

(a) SSS image, (b) seabed bottom characteristics classification map, (c) topographic map, (d) direct terrain inversion result without considering seabed bottom characteristics

Figure 1

The essence of the change of mean backscattering intensity of seafloor with grazing angle is the result of redistribution of sound energy projected to the seafloor in space. How to quantitatively describe the above distribution process is a hot and difficult issue in domestic and international research. The representative research achievements are Lambert model, small roughness perturbation approximate model, Kirchoff model.Approximate models and composite roughness approximate models, etc. (Thorsos, 1989; Jackson, 1986, 1992; Broschat, 1997).Although these models can describe the backscattering of rough seafloor to some extent, none of them is strictly satisfied.That is to say, the observed seafloor backscattering intensity varies with the grazing angle not strictly in accordance with the above model, and rough rock seafloor and discrete targets such as shell debris also invalidate the scattering model, which increases the uncertainty of seabed bottom characteristics classification results.

\section{Classification of seabed bottom characteristics Matter}

At present, seabed bottom characteristics classification research mainly focuses on seabed bottom characteristics-related feature description, classifier design and minimum classification unit setting strategy.Taking SSS as an example, there is no unique correspondence between the seabed bottom characteristics and the texture in the image, so the reliability of classification based only on the texture still needs to be improved.
A large number of studies have shown that there is a strong correlation between the echo intensity distribution and the seabed bottom characteristics, but there is little research on the relationship between them ${ }^{[9,10]}$.In terms of classifier design, the unsupervised classification of seabed bottom characteristics can be implemented by means of image inherent mode. SSS image implies topographic and seabed bottom characteristics information, which usually ignores topographic factors and thus affects the reliability of seabed bottom characteristics classification. Relatively to unsupervised classification, scholars at home and abroad have carried out a lot of research on supervised classification, such as early Bayesian method, current machine learning methods such as self-organizing neural network, support vector machine method, random forest and so on. They also carried out optimum research on initial setting of classifier and collection speed.In terms of minimum classification unit selection, the traditional classification strategy uses pixels or rectangular blocks of fixed size as minimum units to extract feature and classify the base material.Object segmentation has been successfully applied in remote sensing image classification ${ }^{[11]}$. This method is superior to pixel-based classification in determining boundary of seabed bottom characteristics category and overall classification accuracy, and omits the steps of processing classification speckle noise.In addition, foreign scholars have done a lot of research on segmentation steps in object classification methods, put forward descriptive characteristics such as homogeneity and complexity, and carried out in- 
depth research on regional variational segmentation methods. Better classification results have been achieved in different environments There are few studies in this field in China, mostly staying on the segmentation of significant objects.

The above methods for seabed bottom characteristics classification are based on highquality SSS images, but the existing methods are limited due to less research on the reliability reduction of seabed bottom characteristics classification caused by such problems as incomplete correction of radiation sag in the image, strong echo intensity of target upstream surface and shadowing of backwave surface caused by SSS imaging, large variation of echo intensity directly under the trailer, and gray difference between strips.Accurate classification of the seabed bottom characteristics is carried out in the whole area by means of SSS surveying information.

\section{Multi-type subsea observation data fusion}

Seabed bottom characteristics classification by using acoustic equipment such as SSS and multibeam sounding system has become more and more advantageous for quickly identifying the type of seabed bottom characteristics.At present, the classification accuracy of single equipment seabed bottom characteristics is limited, the universality of model algorithm is not strong, and the reliability is poor.Combining the theories and methods of highfrequency subsea acoustics, data fusion and mining, machine learning and other fields, the paper focuses on the correlation between multi-source observation data and seabed bottom characteristics and the complementarity of representing subsea characteristics, multi-source observation data matching fusion algorithm and seabed bottom characteristics hierarchy classification method, builds the spatial matching and fusion model of subsea classification results, improves the seabed bottom characteristics classification map.Spatial resolution and accuracy.

A key factor in the classification of seabed bottom characteristicss using multi-type seafloor observation data is the availability of abundant data sources.This requires simultaneous single-beam, side-sweep sonar and multi-beam measurements. In order to establish a correlation model with the seabed bottom characteristics, it is necessary to know the type of seabed bottom characteristics in the measuring area and to have as many types as possible.At the same time, the data satisfying the above requirements are now easily found. In recent years, more and more attention has been paid to the importance of comprehensive survey and measurement. For example, the accuracy of multibeam depth measurement can be checked and evaluated by using single-beam data, and the shape of submarine obstacles can be reflected in detail by combining side-sweep sonar images with multibeam depth measurement data.Therefore, many measurement tasks require simultaneous side-scan sonar and multi-beam measurements, which ensures the feasibility of application of data sources and seabed bottom characteristics classification methods.

\section{Summary and Prospect}

Our country has made great progress in the research of sonar technology and computer vision level.seabed bottom characteristics classification technology of submarine sonar image also faces some technical challenges in the research.At present, with the vigorous development of artificial intelligence, we need to explore and verify with a large number of sampling and observation data based on the theory of acoustic model, so as to improve the intelligence level of seabed bottom characteristics detection. In the future, we need to make efforts in the following aspects:

(a) Subsea Acoustics Database Construction

In the long-term marine observation, the research results are difficult to compare and share among different research units, involving different sea areas, different data acquisition and processing schemes, different types of sonar and sensors, and lack of data processing and sharing mechanism, resulting in a large number of data resources idle and wasted.The existing subsea observation data should be planned as a whole to promote the construction of data resource sharing platform.

(b) Improve the standardized process of backscattering data

The backscattering intensity must be corrected and compensated to accurately reflect the acoustic characteristics of the seafloor, and other factors affecting the backscattering intensity must be compensated.In addition to the submarine topographic correction, the sound propagation process should be simulated by combining the submarine ground sound model and the scattering model.To clarify the mechanism and correction method of each factor, and gradually improve the standardized processing process and quality control standard of backscattering data.

(c) Deep integration with artificial intelligence theory 
In terms of underwater target recognition, it is very important to improve the accuracy and reliability of underwater target recognition by using deep learning method, to fully integrate the auxiliary function of marine environment information in intelligent analysis and decisionmaking system, to realize the deep combination of artificial intelligence technology and marine detection technology, and to develop intelligent marine space detection technology.

(d) Application of Unmanned Underwater Measuring Platform

With the technical advantages of AUV and other underwater mobile platforms, such as high observation accuracy and complete observation information, real-time seabed bottom characteristics detection and identification technology has already obtained technical foundation.In the future, unmanned underwater platform can also be used to detect deep water subsea strata with high accuracy, thus effectively improving the detection error and reliability in deep water areas.

Correspondence author: JIN Shaohua Fund project: The National Natural Science Fund (41876103)

\section{References}

1. F.G.S. Dr Philippe Blondel C.Geol., PhD., M.Sc. The Handbook of Sidescan Sonar[M].2009.

2. Duncan Tamsett, Jason MclIlvenny, Andrew Watts. Colour sonar: Multi-frequency sidescan images of the seabed in the Inner Sound of the Pentland Firth, Scotland[J]. Journal of Marine Science and Engineering, 2016,4(1): 26.

3. Daniel Buscombe. Shallow water benthic imaging and substrate characterization using recreationalgrade sidescan-sonar[J]. Environmental Modelling and Software, 2017,89:1-18.

4. Li Haisen, Wei Bo, Du Weidong. Research progress of multibeam synthetic aperture sonar [J]. Acta Sinica Sinica, 2017, 46 (10): 1760-1769.

5. Roy Edgar Hansen, Hayden John Callow, Torstein O. Saeboe, et al. Challenges in Seafloor Imaging and Mapping With Synthetic Aperture Sonar[J]. IEEE Transactions on Geoscience \& Remote Sensing, 2011, 49(10):3677-3687.

6. Wang Aixue, Zhang Hongmei, Wang Xiao, et al. Data processing and seamless imaging of side scan sonar [J]. Surveying and mapping geographic information, 2017, 42 (1): 26-29.

7. Jianhu Zhao, Aixue Wang, Hongmei Zhang, et al. Mosaic method of side-scan sonar ship images using corresponding features[J]. Iet Image Processing, 2013, 7(6): 616-623.

8. Jianhu Zhao, Xiaodong Shang, Hongmei Zhang.
Side-Scan Sonar Image Mosaic Using Couple Feature Points with Constraint of Track Line Positions[J]. Remote Sensing, 2018, 10(6): 953

9. Anvri Simkooei Alireza, Snellen Mirjam, Dick $\mathrm{G}$ Simons. Riverbed sediment classification using multi-beam echo-sounder backscatter data[J]. Journal of the Acoustical Society of America, 2009, 126(4): 1724-38.

10. Gilles Le Chenadec, J. M. Boucher, Xavier Lurton Angular Dependence of -Distributed Sonar Data[J]. Geoscience \& Remote Sensing IEEE Transactions on, 2007,45(5): 1224-1235.

11. T.Blaschke. Object based image analysis for remote sensing[J]. Isprs Journal of Photogrammetry \& Remote Sensing, 2010, 65(1):2-16. 